\title{
Événements indésirables à la suite d'une prophylaxie antibiotique de masse pendant une éclosion de streptocoque du groupe A à l'École de leadership et de recrues des Forces canadiennes
}

\author{
Diane Lu ${ }^{1 \star}$, Barbara Strauss ${ }^{1}$, Kristen Simkus ${ }^{1}$, Martin Tepper $^{1}$, François Gagnon², \\ Noémie Johnson², Eric Girard², Kirsten Barnes
}

\section{Résumé}

Contexte : Entre décembre 2016 et mars 2018, deux éclosions d'infections par le streptocoque du groupe A se sont produites à l'École de leadership et de recrues des Forces canadiennes. Un programme volontaire de prophylaxie antibiotique de masse a été mis en œuvre en mars 2018, afin d'interrompre une éclosion de streptocoque du groupe A continuelle et d'empêcher des éclosions à venir.

Méthodes : On a offert aux instructeurs et aux recrues une injection intramusculaire unique de 1,2 million d'unités de pénicilline $G$ benzathine (PGB). Les personnes ayant une allergie à la pénicilline se sont vues offrir de l'azithromycine; $500 \mathrm{mg}$ par voie orale une fois par semaine pendant quatre semaines consécutives. On a également demandé aux instructeurs et aux recrues de remplir un sondage volontaire et anonyme une semaine après l'administration de la prophylaxie antibiotique de masse afin de détecter les événements indésirables liés à cette dernière.

Résultats : La prophylaxie antibiotique de masse a été offerte à 2749 personnes; 2707 d'entre elles ont accepté de la recevoir (98,5\%). La majorité du personnel a connu des événements indésirables dans les jours suivants la prophylaxie antibiotique de masse; $92,3 \%$ des personnes qui ont reçu de la PGB ont signalé une douleur localisée au site d'injection et 70,2 \% des personnes qui ont reçu de l'azithromycine ont signalé des symptômes gastro-intestinaux. Toutefois, on a seulement signalé cinq cas d'événements indésirables graves et moins de $1 \%$ des recrues n'ont pas pu suivre leur formation militaire de base en raison d'événements indésirables liés à la prophylaxie antibiotique de masse.

Conclusion : Le programme de prophylaxie antibiotique de masse mis en œuvre en mars 2018 est le premier du genre dans les Forces armées canadiennes et le plus grand usage de la PGB dans un groupe défini au Canada. II n'a entraîné que très peu d'événements indésirables graves et n'a eu que peu d'incidence sur la réussite de la formation des recrues.

Citation proposée : Lu D, Strauss B, Simkus K, Tepper M, Gagnon F, Johnson N, Girard E, Barnes K. Événements indésirables à la suite d'une prophylaxie antibiotique de masse pendant une éclosion de streptocoque du groupe A à l'École de leadership et de recrues des Forces canadiennes. Relevé des maladies transmissibles au Canada 2020;46(9):297-305. https://doi.org/10.14745/ccdr.v46i09a03f

Mots-clés : éclosions de maladies infectieuses, recrues militaires, Canada, Streptococcus pyogenes, prophylaxie antibiotique de masse, événements indésirables
Cette oeuvre est mise à la disposition selon les termes de la licence internationale Creative Commons Attribution 4.0

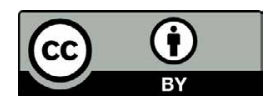

Affiliations

1 Direction de la protection de la santé de la Force, Services de santé des Forces canadiennes, Ottawa, ON

${ }^{2} 41^{\mathrm{e}}$ Centre des Services de santé des Forces canadiennes, Richelain, QC

${ }^{\star}$ Correspondance : diane.lu@forces.gc.ca 


\section{Introduction}

Le streptocoque du groupe A est une bactérie bêta-hémolytique à Gram positif qui provoque des infections qui se présentent habituellement sous forme de pharyngite ou d'amygdalite $(1,2)$. Cependant, il peut causer des maladies graves chez des adultes autrement en bonne santé. Des éclosions de streptocoque du groupe $A$ invasif chez des recrues militaires américaines ont été documentées depuis les années 1940 (3). Les facteurs de risque associés au streptocoque du groupe $A$ invasif dans les milieux militaires comprennent les dortoirs non séparés, l'entraînement dans des conditions stressantes et l'exposition à des contacts étroits (2-7). La prophylaxie antibiotique de masse a déjà été utilisée pour réduire efficacement la maladie provoquée par le streptocoque du groupe $A$ dans les centres de formation militaire américains (3,8-14). Les deux antibiotiques utilisés dans le cadre de la prophylaxie antibiotique de masse sont la pénicilline $G$ benzathine (PGB) administrée par voie intramusculaire et l'azithromycine administrée par voie orale. Parmi les effets indésirables couramment signalés chez les adultes pour la PGB injectée comprennent les réactions locales au site d'injection, y compris la douleur, les éruptions cutanées, les troubles articulaires et les étourdissements (15). Les effets indésirables les plus souvent signalés chez les adultes après l'administration d'azithromycine par voie orale étaient la diarrhée ou les selles molles (de $4 \%$ à $5 \%$ ), les nausées (de $3 \%$ à $4 \%$ ), les douleurs abdominales (de $2 \%$ à $3 \%$ ) et les vomissements (1\%) (16). Toutefois, l'incidence des événements indésirables survenus après l'administration de la prophylaxie antibiotique de masse sur les recrues militaires américaines n'a pas été publiée.

L'École de leadership et de recrues des Forces canadiennes (ELRFC) est l'installation de formation militaire de base (FMB) pour les Forces armées canadiennes (FAC). Chaque année, I'ELRFC offre la FMB à environ 4400 recrues des FAC, dans un établissement qui héberge jusqu'à 1500 recrues à la fois (Maj E. Girard, communication personnelle, le 29 mars 2019). La formation est un programme intensif structuré de dix à douze semaines et toute interruption de la formation peut contribuer à ce qu'une recrue militaire échoue ou doive reprendre le cours. Entre décembre 2016 et mars 2018, il y a eu deux éclosions de streptocoque du groupe A de la souche emm6.4 (17) confirmée en laboratoire.

En raison de ces deux éclosions de streptocoque du groupe $A$ survenues au cours d'une période de dix mois, le Groupe des services de santé des Forces canadiennes, en consultation avec les organismes de santé publique civils régionaux et provinciaux, ont décidé de mettre en œuvre un programme volontaire de prophylaxie antibiotique de masse dans le but d'interrompre l'éclosion et d'empêcher la propagation à venir du streptocoque du groupe A parmi le personnel militaire de l'ELRFC. C'était la première fois qu'un programme de prophylaxie antibiotique de masse pour une éclosion de streptocoque du groupe $A$ a été mis en œuvre chez les FAC. Compte tenu des effets indésirables potentiels des antibiotiques utilisés pour la prophylaxie antibiotique de masse, du manque de documentation publiée sur ses effets sur les recrues militaires et de l'incidence nocive que cela pourrait avoir sur la capacité d'une recrue à réussir la $\mathrm{FMB}$, la présente étude examine les résultats négatifs à court terme vécus par les recrues des FAC et leurs répercussions sur la FMB, à la suite de la prophylaxie antibiotique de masse.

\section{Méthodes}

Un programme de prophylaxie antibiotique de masse volontaire a été mis en œuvre entre le 7 mars et le 28 mai 2018 à l'ELRFC, située à Saint-Jean-sur-Richelieu, au Québec (Canada). On a offert aux instructeurs et aux recrues une injection intramusculaire unique de 1,2 million d'unités de PGB. La PGB a été administrée par voie intramusculaire dans le muscle vastus lateralis du personnel traité du 7 mars au 22 avril 2018. En raison d'une révision de la monographie de produit, la PGB a été administrée au site dorsoglutéal chez le personnel traité du 23 avril au 28 mai 2018. Les personnes qui ont signalé une allergie à la pénicilline ou qui ont refusé la PGB se sont vu offrir de l'azithromycine à une dose de $500 \mathrm{mg}$ par voie orale une fois par semaine pendant quatre semaines consécutives. Les recrues et les instructeurs ont reçu des feuilles d'information sur les médicaments, disponibles dans les deux langues officielles (français et anglais).

Un sondage sur papier, volontaire et anonyme, a été élaboré afin d'effectuer la surveillance des effets indésirables à la suite de la prophylaxie antibiotique de masse. Le sondage était disponible dans les deux langues officielles et comprenait 14 questions non démographiques (annexe). Le sondage a été offert une semaine après l'administration de la PGB, ou une semaine après la première dose orale d'azithromycine. On a demandé aux répondants du sondage de déclarer eux-mêmes le type d'antibiotique reçu, s'ils avaient ressenti l'un des effets secondaires communs énumérés dans les monographies de produit respectives des antibiotiques et si ces effets secondaires limitaient leur participation à la FMB. Les sondages remplis ont été retournés à la Direction de la protection de la santé de la Force par courrier interne. Les données ont été saisies manuellement dans une base de données Access et un échantillon aléatoire de $10 \%$ des sondages $(n=145)$ a été saisi de nouveau afin d'assurer l'exactitude de la saisie des données effectuée par de multiples employés. De plus, une surveillance passive au moyen d'une feuille de calcul formatée en tableau descriptif détaillé a été élaborée pour que les médecins locaux fournissent des renseignements sur le personnel qui a été évalué pour des effets indésirables graves ou inhabituels à la suite de la prophylaxie antibiotique de masse.

\section{Résultats}

Du 7 mars au 28 mai 2018, la prophylaxie antibiotique de masse a été offerte à 2534 recrues et à 215 instructeurs (tableau 1). 
Dans l'ensemble, elle a été administrée à 2707 membres du personnel des FAC (taux de participation de 98,5\%). Sur les 2707 personnes qui ont reçu la prophylaxie antibiotique de masse, 2398 (88,6 \%) ont reçu de la PGB et les 309 autres $(11,2 \%)$ ont reçu de l'azithromycine.

Tableau 1 : Fréquence des recrues et des instructeurs qui acceptent ou refusent la prophylaxie antibiotique de masse à l'École de leadership et de recrues des Forces canadiennes, du 7 mars au 30 avril 2018

\begin{tabular}{|l|r|c|r|r|r|r|r|}
\multirow{2}{*}{$\begin{array}{c}\text { Personnel } \\
\text { ayant reçu le } \\
\text { médicament }\end{array}$} & \multicolumn{2}{|c|}{ PGB } & \multicolumn{2}{c|}{ Azithromycine } & \multicolumn{2}{c|}{ Refus } & \multirow{2}{*}{ Total } \\
\cline { 2 - 8 }$n$ & $\mathbf{n}$ & \multicolumn{1}{c|}{ n } & \multicolumn{1}{c|}{$\%$} & n & $\%$ & \\
\hline Recrue & 2226 & 87,8 & 281 & 11,1 & 27 & 1,1 & 2534 \\
\hline Instructeur & 172 & 80,0 & 28 & 13,0 & 15 & 7,0 & 215 \\
\hline Total & 2398 & 87,2 & 309 & 11,2 & 42 & 1,5 & 2749 \\
\hline
\end{tabular}

Abréviation : $\mathrm{PGB}$, pénicilline $\mathrm{G}$ benzathine

\section{Résultats - surveillance des événements indésirables}

Au cours de la période du 14 mars au 7 mai 2018,

2149 membres du personnel des FAC ont reçu un sondage sur les événements indésirables liés à la prophylaxie antibiotique de masse. Le sondage a été rempli par 1752 personnes (taux de réponse de $81,5 \%$ ), mais 41 répondants ont été exclus de l'analyse en raison de données manquantes (29 n'ont pas indiqué l'antibiotique qu'ils avaient reçu et 12 n'ont pas indiqué s'ils étaient une recrue ou un instructeur). Des 1711 personnes, 41 autres ont été exclues parce qu'elles ont indiqué qu'elles avaient refusé la prophylaxie antibiotique de masse ou qu'elle ne leur avait pas été offerte (tableau 2). Les 1670 répondants restants ont été inclus dans l'analyse des événements indésirables.

Tableau 2 : Nombre d'événements indésirables signalés ${ }^{a}$ à la suite d'une prophylaxie antibiotique de masse à l'intention des recrues et des instructeurs à l'École de leadership et de recrues des Forces canadiennes, du 7 mars au 30 avril 2018

\begin{tabular}{|c|c|c|c|c|c|c|c|}
\hline \multirow{2}{*}{$\begin{array}{c}\text { Personnel } \\
\text { ayant reçu le } \\
\text { médicament }\end{array}$} & \multicolumn{2}{|c|}{ PGB } & \multicolumn{2}{|c|}{ Azithromycine } & \multicolumn{2}{|c|}{$\begin{array}{c}\text { Aucune } \\
\text { PAM }^{\mathrm{b}}\end{array}$} & \multirow{2}{*}{ Total } \\
\hline & n & $\%$ & $\mathbf{n}$ & $\%$ & $\mathbf{n}$ & $\%$ & \\
\hline Recrue & 1358 & 86,6 & 183 & 11,7 & 27 & 1,7 & 1568 \\
\hline Instructeur & 104 & 72,7 & 25 & 17,5 & 14 & 9,8 & 143 \\
\hline Total & 1462 & 85,4 & 208 & 12,2 & 41 & 2,4 & 1711 \\
\hline
\end{tabular}

Des 1670 répondants au sondage, 1462 (87,5\%) ont reçu la PGB. Parmi eux, 1358 (92,9\%) ont signalé au moins un effet indésirable. L'effet indésirable le plus souvent signalé après l'injection de PGB a été une douleur localisée au site d'injection (tableau 3). Vingt recrues et trois instructeurs (i.e. 1,6\% de tous les répondants traités à l'aide de la PGB) ont demandé des soins médicaux pour leur douleur (tableau 3). Les répondants qui ont reçu une injection de PGB dans le muscle vastus lateralis présentaient des risques beaucoup plus élevés de signaler une douleur que ceux qui ont reçu l'injection dans le muscle glutéal $(2,45$; IC à $95 \%: 1,76-3,42 ; p=0,002)$. Quatre répondants recrus ont déclaré avoir échoué la FMB et sept autres ont déclaré avoir dû reprendre le cours à la suite d'un événement indésirable à la suite d'une injection de PGB; ensemble, ces 11 répondants représentent $0,8 \%$ des 1358 répondants qui ont déclaré avoir reçu une injection de PGB. Les recrues (30,6\%; IC à $95 \%$ : $28,2-33,2)$ et les instructeurs $(27,9 \%$; IC à $95 \%$ : 20,1-37,3) ont déclaré avoir éprouvé la douleur le plus souvent au point d'injection pendant trois jours, environ $50 \%$ des recrues et des instructeurs ayant déclaré que la journée de douleur la plus intense était le premier jour suivant l'injection.

Des 1670 répondants du sondage, 208 (12,5\%) ont reçu de l'azithromycine. Parmi eux, 146 (70,2 \%) ont déclaré avoir ressenti au moins un symptôme gastro-intestinal (GI) après leur première dose de l'antibiotique. Les symptômes $\mathrm{Gl}$ signalés comprenaient de la diarrhée, des maux d'estomac et des nausées ou des vomissements (tableau 4). Les symptômes Gl ont été le plus souvent signalés le premier jour après avoir reçu la première dose de l'antibiotique oral (tableau 4). Deux répondants recrus ont déclaré avoir échoué la FMB et un autre a déclaré avoir dû reprendre le cours en raison d'un effet indésirable de l'azithromycine; ensemble, ces trois personnes représentent 1,6\% des 183 répondants recrus qui ont déclaré avoir reçu une dose orale d'azithromycine.

Des 2707 personnes qui ont reçu la prophylaxie antibiotique de masse, il y a eu un total de cinq cas signalés d'événements indésirables graves nécessitant une hospitalisation (quatre recrues et un instructeur), tous après l'injection de PGB en mars 2018. Les cinq effets indésirables comprenaient un cas de chacun des événements suivants : anaphylaxie, syndrome de loge (rhabdomyolyse avec lésion rénale aiguë), cellulite, hématome au point d'injection et vomissements excessifs. La durée de I'hospitalisation variait d'un jour (anaphylaxie, hématome, vomissements excessifs) à plusieurs jours (cellulite, rhabdomyolyse) et les cinq personnes se sont complètement rétablies. 
Tableau 3 : Résumé des effets indésirables autodéclarés et demande d'aide médicale par les recrues et les instructeurs de l'École de leadership et de recrues des Forces canadiennes à la suite de l'injection de pénicilline G benzathine, du 7 mars au 30 avril 2018

\begin{tabular}{|c|c|c|c|c|c|c|c|c|c|c|c|c|}
\hline \multirow{2}{*}{$\begin{array}{l}\text { Autodéclaration } \\
\text { du personnel }\end{array}$} & \multicolumn{2}{|c|}{$\begin{array}{l}\text { Douleur au point } \\
\text { d'injection }\end{array}$} & \multicolumn{2}{|c|}{ Maux de tête } & \multicolumn{2}{|c|}{$\begin{array}{c}\text { Nausées ou } \\
\text { vomissements }\end{array}$} & \multicolumn{2}{|c|}{$\begin{array}{c}\text { Éruptions } \\
\text { cutanées ou } \\
\text { démangeaisons }\end{array}$} & \multicolumn{2}{|c|}{$\begin{array}{l}\text { Vertige or léger } \\
\text { étourdissement }\end{array}$} & \multicolumn{2}{|c|}{$\begin{array}{l}\text { Douleurs } \\
\text { articulaires }\end{array}$} \\
\hline & $\mathbf{n}$ & $\begin{array}{c}(\%, I C \\
\text { à } 95 \%)\end{array}$ & $\mathbf{n}$ & $\begin{array}{l}(\%, I C \\
\text { à } 95 \%)\end{array}$ & $\mathbf{n}$ & $\begin{array}{l}(\%, I C \\
\text { à } 95 \%)\end{array}$ & $\mathbf{n}$ & $\begin{array}{c}(\%, I C \\
\text { à } 95 \%)\end{array}$ & $\mathbf{n}$ & $\begin{array}{l}(\%, I C \\
\text { à } 95 \%)\end{array}$ & $\mathbf{n}$ & $\begin{array}{c}(\%, I C \\
\text { à } 95 \%)\end{array}$ \\
\hline Recrues & 1248 & $\begin{array}{r}(92,2 \\
90,7-93,5)\end{array}$ & 225 & $\begin{array}{r}(17,4 \\
15,4-19,5)\end{array}$ & 96 & $\begin{array}{r}(7,5 \\
6,2-9,1)\end{array}$ & 84 & $\begin{array}{r}(6,6 \\
5,3-8,1)\end{array}$ & 223 & $\begin{array}{r}(17,3 \\
15,4-19,5)\end{array}$ & 298 & $\begin{array}{r}(22,7 \\
20,5-25,0)\end{array}$ \\
\hline Instructeurs & 98 & $\begin{array}{r}(94,2 \\
88,0-97,3)\end{array}$ & 16 & $\begin{array}{r}(15,8 \\
10,0-24,2)\end{array}$ & 4 & $\begin{array}{r}(4,0 \\
1,6-9,9)\end{array}$ & 4 & $\begin{array}{r}(4,1 \\
1,6-10,1)\end{array}$ & 12 & $\begin{array}{r}(11,9 \\
6,9-19,6)\end{array}$ & 21 & $\begin{array}{r}(20,6, \\
13,9-29,4)\end{array}$ \\
\hline Total & 1346 & $\begin{array}{r}(92,3 \\
90,8-93,6)\end{array}$ & 241 & $\begin{array}{r}(17,3 \\
15,4-19,3)\end{array}$ & 100 & $\begin{array}{r}(7,3 \\
6,0-8,8)\end{array}$ & 88 & $\begin{array}{r}(6,4 \\
5,2-7,8)\end{array}$ & 235 & $\begin{array}{r}(16,9 \\
15,1-19,0)\end{array}$ & 319 & $\begin{array}{r}(22,5 \\
20,4-24,8)\end{array}$ \\
\hline $\begin{array}{l}\text { Nombre de jours } \\
\text { de la durée de } \\
\text { l'événement } \\
\text { indésirable }^{a}\end{array}$ & & 3 & & 1 & & 1 & & 1 & & 1 & & 2 \\
\hline $\begin{array}{l}\text { Recrues - } \\
\text { Demande d'aide } \\
\text { médicale à la suite } \\
\text { d'un événement } \\
\text { indésirable }\end{array}$ & 20 & $\begin{array}{r}(1,7 \\
1,1-2,6)\end{array}$ & 8 & $\begin{array}{r}(4,0 \\
2,0-7,8)\end{array}$ & 5 & $\begin{array}{r}(5,7 \\
2,3-13,1)\end{array}$ & 6 & $\begin{array}{r}(9,5 \\
4,2-20,0)\end{array}$ & 15 & $\begin{array}{r}(7,7 \\
4,7-12,4)\end{array}$ & 13 & $\begin{array}{r}(5,1 \\
2,9-8,5)\end{array}$ \\
\hline $\begin{array}{l}\text { Instructeurs - } \\
\text { Demande d'aide } \\
\text { médicale à la suite } \\
\text { d'un événement } \\
\text { indésirable }\end{array}$ & 3 & $\begin{array}{r}(3,1, \quad 1,0- \\
9,3)\end{array}$ & 0 & $(0,0,0)$ & 0 & $(0,0,0)$ & 0 & $(0,0,0)$ & 0 & $(0,0,0)$ & 1 & $\begin{array}{r}(5,3 \\
0,7-30.7)\end{array}$ \\
\hline $\begin{array}{l}\text { Recrues déclarant } \\
\text { avoir reçu un } \\
\text { congé de maladie }\end{array}$ & $\begin{array}{r}16 \text { sur } \\
18\end{array}$ & $\begin{array}{r}(88,9 \\
61,1-97,6)\end{array}$ & $\begin{array}{r}2 \text { sur } \\
7\end{array}$ & $\begin{array}{r}(28,6 \\
4,2-78,5)\end{array}$ & $\begin{array}{r}2 \text { sur } \\
3\end{array}$ & $\begin{array}{r}(66,7 \\
20,8-93,9)\end{array}$ & $\begin{array}{r}2 \text { sur } \\
4\end{array}$ & $\begin{array}{r}(50, \\
15,0-85,0)\end{array}$ & $\begin{array}{rr}6 & \text { sur } \\
11\end{array}$ & $\begin{array}{r}(54,5 \\
22,6-83,2)\end{array}$ & $\begin{array}{rr}7 \text { sur } \\
11\end{array}$ & $\begin{array}{r}(63,6 \\
28,8-88,3)\end{array}$ \\
\hline
\end{tabular}

a Nombre le plus souvent déclaré de jours de réactions indésirables après la prophylaxie antibiotique de masse (recrues et instructeurs)

Tableau 4 : Résumé des effets indésirables autodéclarés et demande d'aide médicale à la suite de I'administration de l'azithromycine ${ }^{a}$ par les membres du personnel de l'École de leadership et de recrues des Forces canadiennes, du 7 mars au 30 avril 2018

\begin{tabular}{|c|c|c|c|c|c|c|}
\hline \multirow[t]{2}{*}{$\begin{array}{l}\text { Autodéclaration } \\
\text { du personnel }\end{array}$} & \multicolumn{2}{|c|}{ Diarrhée } & \multicolumn{2}{|c|}{ Maux d'estomac } & \multicolumn{2}{|c|}{$\begin{array}{c}\text { Nausées ou } \\
\text { vomissements }\end{array}$} \\
\hline & n & $\begin{array}{c}(\%, \text { IC à } \\
95 \%)\end{array}$ & $\mathrm{n}$ & $\begin{array}{l}(\%, \text { IC à } \\
95 \%)\end{array}$ & $\mathbf{n}$ & $\begin{array}{l}(\%, \text { IC à } \\
95 \%)\end{array}$ \\
\hline Recrues & 78 & $\begin{array}{r}(47,3 \\
39,8-54,9)\end{array}$ & 91 & $\begin{array}{r}(53,2 \\
45,8-60,5) \\
\end{array}$ & 57 & $\begin{array}{r}(35,0 \\
28,1-42,6)\end{array}$ \\
\hline Instructeurs & 14 & $\begin{array}{r}(60,9 \\
40,8-77,8)\end{array}$ & 15 & $\begin{array}{r}(65,2 \\
44,9-81,2)\end{array}$ & 5 & $\begin{array}{r}(25,0 \\
11,2-46,9)\end{array}$ \\
\hline Total & 92 & $\begin{array}{r}(48,9 \\
41,9-56,0) \\
\end{array}$ & 106 & $\begin{array}{r}(54,6 \\
47,6-61,5) \\
\end{array}$ & 62 & $\begin{array}{r}(33,9 \\
27,4-41,0) \\
\end{array}$ \\
\hline $\begin{array}{l}\text { Recrues - } \\
\text { Demande d'aide } \\
\text { médicale à la suite } \\
\text { d'un événement } \\
\text { indésirable }\end{array}$ & 1 & $\begin{array}{r}(1,4 \\
0,2-7,4)\end{array}$ & 2 & $\begin{array}{r}(2,4 \\
0,7-8,2)\end{array}$ & 3 & $\begin{array}{r}(5,3, \\
1,8-14,4)\end{array}$ \\
\hline $\begin{array}{l}\text { Instructeurs - } \\
\text { Demande d'aide } \\
\text { médicale à la suite } \\
\text { d'un événement } \\
\text { indésirable }\end{array}$ & & 0 & & 0 & & 0 \\
\hline $\begin{array}{l}\text { Recrues déclarant } \\
\text { avoir reçu un } \\
\text { congé de maladie }\end{array}$ & Aucune & $(0,0)$ & $\begin{array}{r}1 \text { sur } \\
2\end{array}$ & $\begin{array}{r}(50,0 \\
9,5-90,6)\end{array}$ & $\begin{array}{r}2 \text { sur } \\
3\end{array}$ & $\begin{array}{r}(66,7 \\
20,8-93,9)\end{array}$ \\
\hline
\end{tabular}

Azithromycine administrée sous forme d'une dose orale - la première des quatre doses dans le cadre d'un régime de traitement complet

\section{Discussion}

\section{Résumé des constatations}

En mars 2018, un programme de prophylaxie antibiotique de masse volontaire a été mis en œuvre à l'ELRFC. D'après les sondages autodéclarés, la majorité du personnel a connu des événements indésirables au cours de la première semaine suivant la prophylaxie antibiotique de masse; 92,3\% du personnel qui a reçu une injection de PGB a signalé une douleur au site d'injection et 70,2 \% des personnes qui ont reçu de l'azithromycine par voie orale ont signalé des symptômes Gl après leur première dose. Cependant, peu de ces effets indésirables semblent avoir eu des répercussions importantes sur le personnel. Seulement cinq incidents indésirables graves ont été signalés au cours du premier mois suivant la prophylaxie antibiotique de masse et moins de $1 \%$ des recrues ont déclaré ne pas être en mesure de terminer la FMB à la suite de la prophylaxie antibiotique de masse.

\section{Comparaison avec la documentation}

Les effets indésirables signalés à la suite de la prophylaxie antibiotique de masse sont conformes à ceux cités dans les monographies du médicament, y compris les deux effets indésirables les plus souvent signalés : douleurs localisées à la 
suite de l'injection de PGB et des symptômes GI à la suite d'une dose par voie orale d'azithromycine $(15,16)$. II n'y a eu aucun nouvel événement indésirable inconnu signalé par les recrues pour l'un ou l'autre des antibiotiques. Seulement $0,8 \%$ des recrues des $F A C$ qui ont reçu la PGB ont signalé un événement indésirable qui les a amenées à échouer ou à reprendre la FMB.

Étonnamment, les symptômes GI signalés par les recrues militaires qui ont pris de l'azithromycine étaient d'une magnitude dix fois plus élevée que ceux cités dans la monographie (16), ce qui peut se traduire par un biais de rappel, surtout parce que le sondage a été effectué une semaine après la prophylaxie antibiotique de masse. Toutefois, l'incidence sur la formation des recrues était minime puisque seulement $1,6 \%$ des recrues qui ont reçu de l'azithromycine ont déclaré avoir échoué ou avoir dû reprendre la FMB, ce qui est comparable aux effets indésirables graves ou potentiellement mortels signalés (16).

Les taux d'effets indésirables graves ont été associés à la PGB injectée et administrée seulement au cours du premier mois de la prophylaxie antibiotique de masse (du 7 mars au 22 avril) $(0,15 \%)$, ce qui est également conforme aux études précédentes. Par exemple, sur les 2398 personnes auxquelles on a injecté de la $P G B$, une seule a eu une réaction anaphylactique ( $n=1 / 2398,0,04 \%$ ). De même, dans une étude américaine de 199862 patients suivis pendant les 14 premiers jours suivant l'administration de la pénicilline, $0,05 \%$ ont eu une réaction allergique grave (18). Au cours des 20 dernières années, aucun cas d'anaphylaxie associé à la chimioprophylaxie n'a été signalé dans les centres de formation militaire américains (9).

\section{Points forts et points faibles}

À la suite de la mise en œuvre de la prophylaxie antibiotique de masse à l'ELRFC, il n'y a pas eu de nouveaux cas de streptocoque du groupe $A$ invasif ou de streptocoque du groupe $A$ grave et il y a eu une diminution précipitée du nombre d'écouvillons de gorge positifs au streptocoque du groupe $A$. Cependant, il y a toujours eu des éclosions d'adénovirus parmi les recrues militaires. Ce programme est maintenant en vigueur tout au long de l'année, ce qui a des répercussions sur les ressources en personnel pour l'administration et le calendrier d'administration massive d'antibiotiques, afin de réduire au minimum son incidence sur la FMB.

\section{Répercussions}

Bien qu'aucun effet indésirable grave associé à l'azithromycine n'ait été signalé dans cette intervention, la PGB demeure le choix préférable de chimioprophylaxie par rapport à l'azithromycine, pour plusieurs raisons. Ces raisons comprennent des préoccupations au sujet de la résistance du streptocoque du groupe A à l'azithromycine $(19,20)$ et d'autres agents pathogènes, comme Streptococcus pneumoniae (21), I'arrêt cardiaque dû à l'allongement de l'intervalle OT (22) et la conformité accrue avec le schéma posologique de la PGB par rapport aux multiples doses requises pour l'azithromycine par voie orale (6). On a mis en œuvre un traitement d'observation directe dans les centres de formation militaire américains en raison de la nonconformité liée à cet antibiotique oral.

En raison de la forte prévalence de la douleur autodéclarée au site d'injection de la PGB, les dirigeants ont reporté les tests d'aptitude obligatoires pour les 24 premières heures après l'injection afin de réduire au minimum l'exacerbation de la douleur au site d'injection local (Maj E. Girard, communication personnelle, le 29 mars 2019).

\section{Limites du sondage}

Il est à noter que, compte tenu du besoin urgent de mettre en œuvre la prophylaxie antibiotique de masse pendant l'éclosion de streptocoque du groupe A à l'ELRFC, le sondage sur les effets indésirables n'a pas pu être validé avant son administration. Certaines questions du sondage n'étaient donc peut-être pas claires pour les répondants. Par exemple, les recrues et les instructeurs ont déclaré ne pas avoir demandé de soins médicaux, mais avoir été dispensés de leurs fonctions en raison d'événements indésirables liés à la prophylaxie antibiotique de masse. C'est contradictoire, parce que le personnel militaire ne peut être dispensé de ses fonctions que s'il est évalué en clinique. De plus, l'information comme le sexe et l'âge n'a pas été obtenue au cours du sondage, de sorte que l'on n'a pas été en mesure de déterminer s'il y avait des différences dans les effets indésirables en raison des différences démographiques.

De plus, les répondants au sondage peuvent s'être rappelé de façon incorrecte le type d'antibiotique reçu. Dans le cadre de la $F M B$, les recrues reçoivent souvent un certain nombre de vaccins, ce que les répondants auraient pu confondre avec la PGB injectable. En raison de la nature anonyme du sondage sur les effets indésirables, il a été impossible de confirmer l'exactitude des données autodéclarées sur le type d'antibiotique administré.

Enfin, les injections de PGB ont été administrées par le personnel clinique des FAC, mais les doses d'azithromycine ont été autoadministrées pendant quatre semaines consécutives. On n'a pas observé des membres du personnel auxquels on a prescrit d'azithromycine prendre son antibiotique et la conformité est donc inconnue. De plus, le sondage sur les effets indésirables n'a été distribué qu'une semaine après la première dose d'azithromycine. Il est donc possible que le sondage ait sous-estimé le risque réel d'événements indésirables associés à l'utilisation de l'azithromycine comme prophylaxie pour traiter le streptocoque du groupe $A$.

\section{Conclusion}

L'intervention décrite dans le présent document est la première fois que la prophylaxie antibiotique de masse a été mis en œuvre chez les FAC et il s'agit de la plus grande utilisation de la PGB dans un groupe défini au Canada. L'intervention qui a interrompu l'éclosion de streptocoque du groupe $\mathrm{A}$ était généralement bien tolérée par les recrues et les instructeurs, avec une incidence minimale sur la capacité des recrues à terminer leur FMB. Des effets indésirables communs ont été signalés, comme prévu, 
avec l'utilisation de PGB et d'azithromycine, bien que les effets indésirables graves soient rares. Ainsi, la prophylaxie antibiotique de masse peut être utilisée de façon sécuritaire pour réduire la propagation du streptocoque du groupe A parmi le personnel des FAC et protéger les recrues contre les infections graves au streptocoque du groupe A.

\section{Déclaration des auteurs}

D. L. - Conceptualisation du manuscrit, de l'ébauche originale, de l'examen et de la révision du manuscrit

B. S. - Conservation des données, analyse formelle, examen et révision du manuscrit

K. S. - Conceptualisation et rédaction du sondage, conservation des données, analyse formelle, examen et révision du manuscrit M. T. - Examen et révision du manuscrit

F. G. - Mise en forme et distribution du sondage, examen et révision du manuscrit

N. J. - Distribution du sondage, examen et révision du

manuscrit

E. G. - Examen et révision du manuscrit

K. B. - Examen et révision du manuscrit

\section{Intérêts concurrents}

Présenté précédemment sous forme de présentation sur le podium au Forum 2018 de l'Institut canadien de recherche sur la santé des militaires et des vétérans (ICRSMV), à Regina (Saskatchewan), le 15 octobre 2018.

Avertissement : Les opinions exprimées ne sont que celles des auteurs et ne présentent pas la politique ou la position officielle des Forces armées canadiennes ou du gouvernement du Canada.

\section{Remerciements}

On tient à remercier le $\mathrm{D}^{\mathrm{re}} \mathrm{M}$. St. Amour pour sa contribution à cette enquête sur l'éclosion. De plus, on tient à souligner les efforts de collaboration de tout le personnel clinique du 41 Centre des Services de santé des Forces canadiennes, à Saint-Jean-sur-Richelieu, qui a administré la prophylaxie antibiotique de masse et le sondage sur les effets indésirables. Enfin, merci au personnel d'épidémiologie qui a saisi les données du sondage sur les effets indésirables, le $D^{r} F$. Thériault et le $D^{r e} \mathrm{H}$. McCuaig Edge, pour avoir examiné ce manuscrit et S. Carlucci, pour son aide dans la rédaction des références.

\section{Financement}

Aucun.

\section{Références}

1. Levinson W. Review of Medical Microbiology \& Immunology. $12^{\text {th }}$ ed. New York: McGraw-Hill Medical; 2012.

2. Heymann DL. Streptococcal diseases. In: Heymann DL, editor. Control of Communicable Disease Manual. 20 $0^{\text {th }}$ ed. Washington DC: American Public Health Association; 2015. p 581-9

3. Bernstein $\mathrm{SH}$, Feldman HA, Harper OF Jr, Klingensmith WH. Massoral penicillin prophylaxis in control of streptococcal disease. AMA Arch Intern Med 1954;93(6):894-8. DOI PubMed

4. Sanchez JL, Cooper MJ, Myers CA, Cummings JF, Vest KG, Russell KL, Sanchez JL, Hiser MJ, Gaydos CA. Respiratory infections in the U.S. Military: recent experience and control. Clin Microbiol Rev 2015;28(3):743-800. DOI PubMed

5. Lee SE, Eick A, Ciminera P. Respiratory disease in Army recruits: surveillance program overview, 1995-2006. Am J Prev Med 2008;34(5):389-95. DOI PubMed

6. Crum NF, Russell KL, Kaplan EL, Wallace MR, Wu J, Ashtari P, Morris DJ, Hale BR. Pneumonia outbreak associated with group a Streptococcus species at a military training facility. Clin Infect Dis 2005;40(4):511-8. DOI PubMed

7. Gray GC, Callahan JD, Hawksworth AW, Fisher CA, Gaydos JC. Respiratory diseases among U.S. military personnel: countering emerging threats. Emerg Infect Dis 1999;5(3):379-87. DOl PubMed

8. Lazar HP, Maas GI, Harrison W, Hammond JH, Rantz LA. Streptococcal disease and rheumatic fever in Air Force recruits. II. Prophylaxis with tandem oral penicillin. AMA Arch Intern Med 1957;100(4):614-9. DOI PubMed

9. Webber BJ, Kieffer JW, White BK, Hawksworth AW, Graf PC, Yun HC. Chemoprophylaxis against group A streptococcus during military training. Prev Med 2019;118:142-9. DOI PubMed

10. Schreier AJ, Hockett VE, Seal JR. Mass prophylaxis of epidemic streptococcal infections with benzathine penicillin G.I. Experience at a naval training center during the winter of 1955-56. N Engl J Med 1958;258(25):1231-8. DOI PubMed

11. McFarland RB, Colvin VG, Seal JR. Mass prophylaxis of epidemic streptococcal infections with benzathine penicillin G.-Experience at a naval training center during the winter of 1956-57. N Engl J Med 1958 Jun;258(26):1277-84. DOI PubMed

12. Basiliere JL, Bistrong HW, Spence WF. Streptococcal pneumonia. Recent outbreaks in military recruit populations. Am J Med 1968;44(4):580-9. DOI PubMed

13. Thomas RJ, Conwill DE, Morton DE, Brooks TJ, Holmes CK, Mahaffey WB. Penicillin prophylaxis for streptococcal infections in United States Navy and Marine Corps recruit camps, 1951-1985. Rev Infect Dis 1988;10(1):125-30. DOI PubMed 
14. Centers for Disease Control (CDC). Group A beta-hemolytic streptococcal pharyngitis among U.S. Air Force trainees-Texas, 1988-89. MMWR Morb Mortal Wkly Rep 1990;39(1):11-3. PubMed

15. Penicillin G benzathine drug monograph (accédé 2019-0305). https://www.pfizer.ca/sites/g/files/g10050796/f/201710/ Bicillin_PM.pdf

16. Azithromycin drug monograph (accédé 2019-03-05). https://www.sandoz.ca/sites/www.sandoz.ca/files/ Sandoz\%20Azithromycin\%20PM_EN.pdf

17. Hammond-Collins K, Strauss B, Barnes K, Demczuk W, Domingo MC, Lamontagne MC, Lu D, Martin I, Tepper M, Group A. Group A Streptococcus Outbreak in a Canadian Armed Forces Training Facility. Mil Med 2019;184(3-4):e197-204. DOI PubMed

18. Johannes CB, Ziyadeh N, Seeger JD, Tucker E, Reiter C, Faich $\mathrm{G}$. Incidence of allergic reactions associated with antibacterial use in a large, managed care organisation. Drug Saf 2007;30(8):705-13. DOI PubMed
19. Barrozo CP, Russell KL, Smith TC, Hawksworth AW, Ryan MA, Gray GC. National Department of Defense surveillance data for antibiotic resistance and emm gene types of clinical group A streptococcal isolates from eight basic training military sites. J Clin Microbiol 2003;41(10):4808-11. DOI PubMed

20. Metzgar D, McDonough EA, Hansen CJ, Blaesing CR, Baynes D, Hawksworth AW, Blair PJ, Faix DJ, Russell KL. Local changes in rates of group A Streptococcus disease and antibiotic resistance are associated with geographically widespread strain turnover events. Virulence 2010;1(4): 247-53. DOI PubMed

21. Gray GC, Witucki PJ, Gould MT, Bell SJ, Hiliopoulos KM, McKeehan JA, Fuller JM, Barrozo CP, Hudspeth MK, Smith TC, Ledbetter EK, Wallace MR. Randomized, placebo-controlled clinical trial of oral azithromycin prophylaxis against respiratory infections in a high-risk, young adult population. Clin Infect Dis 2001;33(7):983-9. DOI PubMed

22. Goldstein EJC, Jr Owens RC, Nolin TD. Antimicrobial-associated QT interval prolongation: pointes of interest. Clin Infect Dis 2006;43(12):1603-11. DOI PubMed 


\section{Annexe : Sondage des effets indésirables - prophylaxie antibiotique à l'École de leadership et de recrues des Forces canadiennes}

\section{Introduction:}

Lors des dernières semaines, on pourrait vous avoir offert un de deux médicaments (antibiotiques) pour prévenir de contracter une infection appelée le Streptocoque du groupe A. En répondant à ces quelques questions, nous aimerions savoir si vous avez ressenti certains effets secondaires de cette médication. Veuillez noter que ce questionnaire est volontaire et anonyme.

\section{Instructions:}

Veuillez encercler la réponse qui s'applique le mieux à vous.

Lorsque complété, veuillez remettre ce formulaire à votre instructeur-cadre.

\section{PARTIE A - ANTIBIOTIQUES}

1. Quel antibiotique avez-vous reçu?
a. Bicilline (injection)
b. Azithromycine (pillule)
c. Aucun (Refus ou aucun choix offert)
d. Incertain
[Ne complétez que la partie B]
[Ne complétez que la partie $\mathrm{C}]$
[CESSER le sondage]
[CESSER le sondage]

Douleur au site d'injection

\section{PARTIE B - BICILLINE (INJECTION)}

2. Pendant combien de jours avez-vous ressenti de la douleur au site de l'injection?

\begin{tabular}{|c|c|c|c|c|c|c|}
\hline Pas de douleur & 1 jour & 2 jours & 3 jours & 4 jours & 5 jours & 6 jours ou plus \\
\hline
\end{tabular}

3. Quel jour la douleur a-t-elle été le plus intense?

\begin{tabular}{|l|c|c|c|c|c|c|c|}
\hline $\begin{array}{l}\text { Pas de } \\
\text { douleur }\end{array}$ & $\begin{array}{c}\text { Le jour } \\
\text { même }\end{array}$ & $\begin{array}{c}1 \text { jour } \\
\text { après }\end{array}$ & $\begin{array}{c}2 \text { jours } \\
\text { après }\end{array}$ & $\begin{array}{c}3 \text { jours } \\
\text { après }\end{array}$ & $\begin{array}{c}4 \text { jours } \\
\text { après }\end{array}$ & $\begin{array}{c}5 \text { jours } \\
\text { après }\end{array}$ & $\begin{array}{c}6 \text { jours ou plus } \\
\text { après }\end{array}$ \\
\hline
\end{tabular}

4. Avez-vous consulté à la clinique médicale pour de la douleur au site de l'injection? Pas de douleur Oui Non

5. Si "Oui" à la question 4 , à quelle journée avez-vous consulté à la clinique médicale?

Le jour même

\begin{tabular}{c|c|c|c|c|}
1 jour & 2 jours & 3 jours & 4 jours & 5 jours \\
après & après & après & après & après \\
\hline
\end{tabular}

6 jours ou plus

6. Avez-vous obtenu une note du SSFC (chit) avec des limitations médicales (restrictions des tâches à I'horaire du camp de recrues) en raison de la douleur au site d'injection?
Pas de douleur
Oui
Non

Autres symptômes

7. Avez-vous ressenti un des symptômes suivants suite à l'injection?

Dans l'affirmative, indiquez combien de temps vous avez ressenti ce symptôme.

\begin{tabular}{|c|c|c|c|c|c|c|c|}
\hline Symptômes & $\begin{array}{c}\text { Non- } \\
\text { ressenti }\end{array}$ & $\begin{array}{c}\mathbf{1} \text { jour ou } \\
\text { moins }\end{array}$ & $\begin{array}{c}\mathbf{2} \\
\text { jours }\end{array}$ & $\begin{array}{c}\mathbf{3} \\
\text { jours }\end{array}$ & $\begin{array}{c}\mathbf{4} \\
\text { jours }\end{array}$ & $\begin{array}{c}\mathbf{5} \\
\text { jours }\end{array}$ & $\begin{array}{c}\mathbf{6} \text { jours } \\
\text { ou plus }\end{array}$ \\
\hline Mal de tête & $\mathrm{O}$ & $\mathrm{O}$ & $\mathrm{O}$ & $\mathrm{O}$ & $\mathrm{O}$ & $\mathrm{O}$ & $\mathrm{O}$ \\
\hline Nausée ou vomissement & $\mathrm{O}$ & $\mathrm{O}$ & $\mathrm{O}$ & $\mathrm{O}$ & $\mathrm{O}$ & $\mathrm{O}$ & $\mathrm{O}$ \\
\hline Démangeaison ou rougeur & $\mathrm{O}$ & $\mathrm{O}$ & $\mathrm{O}$ & $\mathrm{O}$ & $\mathrm{O}$ & $\mathrm{O}$ & $\mathrm{O}$ \\
\hline Étourdissement ou faiblesse & $\mathrm{O}$ & $\mathrm{O}$ & $\mathrm{O}$ & $\mathrm{O}$ & $\mathrm{O}$ & $\mathrm{O}$ & $\mathrm{O}$ \\
\hline Douleur articulaire ou osseuse & $\mathrm{O}$ & $\mathrm{O}$ & $\mathrm{O}$ & $\mathrm{O}$ & $\mathrm{O}$ & $\mathrm{O}$ & $\mathrm{O}$ \\
\hline
\end{tabular}


8. Avez-vous consulté à la clinique médicale pour un de ces symptômes?

\begin{tabular}{|c|c|c|c|}
\hline Symptômes & Non-ressenti & Oui & Non \\
\hline Mal de tête & O & O & O \\
\hline Nausée ou vomissement & $\mathrm{O}$ & $\mathrm{O}$ & $\mathrm{O}$ \\
\hline Démangeaison ou rougeur & $\mathrm{O}$ & $\mathrm{O}$ & $\mathrm{O}$ \\
\hline Étourdissement ou faiblesse & $\mathrm{O}$ & $\mathrm{O}$ & $\mathrm{O}$ \\
\hline Douleur articulaire ou osseuse & $\mathrm{O}$ & $\mathrm{O}$ & $\mathrm{O}$ \\
\hline
\end{tabular}

9. Avez-vous obtenu une note du SSFC (chit) avec des limitations médicales (restrictions des tâches à l'horaire du camp de recrues) pour un de ces symptômes?

\begin{tabular}{|c|c|c|c|}
\hline Symptômes & Non-ressenti & Oui & Non \\
\hline Mal de tête & O & O & O \\
\hline Nausée ou vomissement & 0 & 0 & 0 \\
\hline Démangeaison ou rougeur & O & O & 0 \\
\hline Étourdissement ou faiblesse & O & O & 0 \\
\hline Douleur articulaire ou osseuse & O & O & 0 \\
\hline
\end{tabular}

10. Avez-vous échoué ou dû reprendre l'entraînement des recrues en raison des effets secondaires de la bicilline (injection)?

\begin{tabular}{|c|c|c|c|c|c|c|c|}
\hline Non & \multicolumn{3}{|c|}{ Échec } & \multicolumn{4}{|c|}{ Reprise } \\
\hline \multicolumn{8}{|c|}{ PARTIE C - AZITHROMYCINE (PILLULE) } \\
\hline \multicolumn{8}{|c|}{$\begin{array}{l}\text { 11. Avez-vous ressenti un des symptômes suivants après avoir pris une dose (pillule) d'azithromycine? } \\
\text { Dans l'affirmative, indiquez pour combien de jours vous l'avez ressenti. }\end{array}$} \\
\hline Symptômes & $\begin{array}{l}\text { Non- } \\
\text { ressenti }\end{array}$ & $\begin{array}{l}1 \text { jour ou } \\
\text { moins }\end{array}$ & $\begin{array}{c}2 \\
\text { jours }\end{array}$ & $\begin{array}{c}3 \\
\text { jours }\end{array}$ & $\begin{array}{c}4 \\
\text { jours }\end{array}$ & $\begin{array}{c}5 \\
\text { jours }\end{array}$ & $\begin{array}{l}6 \text { jours } \\
\text { ou plus }\end{array}$ \\
\hline Diarrhée & $\mathrm{O}$ & $\mathrm{O}$ & $\mathrm{O}$ & $\mathrm{O}$ & $\mathrm{O}$ & $\mathrm{O}$ & 0 \\
\hline Crampes d'estomac & $\mathrm{O}$ & $\mathrm{O}$ & $\mathrm{O}$ & $\mathrm{O}$ & $\mathrm{O}$ & $\mathrm{O}$ & $\mathrm{O}$ \\
\hline Nausée ou vomissement & $\mathrm{O}$ & $\mathrm{O}$ & $\mathrm{O}$ & $\mathrm{O}$ & $\mathrm{O}$ & $\mathrm{O}$ & $\mathrm{O}$ \\
\hline
\end{tabular}

12. Avez-vous consulté à la clinique médicale pour un de ces symptômes?

\begin{tabular}{|c|c|c|c|}
\hline Symptômes & Non-ressenti & Oui & Non \\
\hline Diarrhée & O & O & O \\
\hline Crampes d'estomac & 0 & 0 & 0 \\
\hline Nausée ou vomissement & 0 & 0 & 0 \\
\hline
\end{tabular}

13. Avez-vous obtenu une note du SSFC (chit) avec des limitations médicales (restrictions des tâches à l'horaire du camp de recrues) pour un de ces symptômes?

\begin{tabular}{|c|c|c|c|}
\hline Symptômes & Non-ressenti & Oui & Non \\
\hline Diarrhée & O & 0 & 0 \\
\hline Crampes d'estomac & 0 & 0 & 0 \\
\hline Nausée ou vomissement & 0 & 0 & 0 \\
\hline
\end{tabular}

14. Avez-vous échoué ou dû reprendre l'entraînement des recrues en raison des effets secondaires de l'azithromycine (pillule)?

\begin{tabular}{|c|c|c|}
\hline Non & Échec & Reprise \\
\hline
\end{tabular}

Consultez un médecin si vous avez des inquiétudes au sujet de ces médicaments.

Maj François Gagnon, MD, Médecin-chef, 41e C Svc S FC St-Jean, (450) 358-7099 poste 6236 